\title{
Asymmetric and Enantiomer-Selective Polymerization of Phenyl-2-pyridyl-o-tolylmethyl Methacrylate
}

\author{
Eiji YASHIMA, ${ }^{*}$ Yoshio OKAMOTO, ${ }^{* *}$ and Koichi HATADA \\ Department of Chemistry, Faculty of Engineering Science, \\ Osaka University, Toyonaka, Osaka 560, Japan
}

(Received December 8, 1986)

\begin{abstract}
The enantiomer-selective (asymmetric selective or stereoelective) polymerization of a bulky racemic monomer phenyl-2-pyridyl-o-tolylmethyl methacrylate (PPyTMA) was investigated with chiral anionic initiators, the complexes of 2,3-dimethoxy-1,4-bis(dimethylamino)butane (DDB) with fluorenyllithium or $N, N^{\prime}$-diphenylethylenediamine monolithium amide, in toluene at $-78^{\circ} \mathrm{C}$. ( - )-Isomer was consumed preferentially over (+)-isomer when (-)DDB was used as a chiral ligand and a highly isotactic polymer was obtained. The initiators showed no enantiomer selectivity in the polymerization of less bulky racemic methacrylates, such as 1-phenylethyl methacrylate and 1,2-diphenylethyl methacrylate which afforded atactic polymers. Enantiomer selectivity increased with conversion up to about $25 \%$. The e.e. of the polymerized monomer at a stage of about $25 \%$ conversion was nearly $100 \%$. This was ascribed to a rigid onehanded helical conformation of highly isotactic poly(PPyTMA) at the early stage of the polymerization. The selectivity did not decrease in the asymmetric copolymerization with triphenylmethyl methacrylate (TrMA), but greatly decreased in the copolymerization with the less bulky methacrylates. The former copolymerization formed a coisotactic polymer and the latter atactic copolymers.
\end{abstract}

KEY WORDS Asymmetric Polymerization / Enantiomer-Selective Polymerization / Anionic Polymerization / Phenyl-2-pyridyl-o-tolylmethyl Methacrylate / (-)-(2R,3R)-2,3-Dimethoxy-1,4-bis(dimethylamino)butane / Optically Active Polymer / Enantiomer Selection / Helix /

Bulky methacrylates such as triphenylmethyl methacrylate (TrMA), diphenyl-2-pyridylmethyl methacrylate (D2PyMA), and diphenyl-4-pyridylmethyl methacrylate (D4PyMA) are unique monomers which form highly isotactic polymers in the anionic polymerization with $n$-butyllithium ( $n$-BuLi) not only in toluene but also in tetrahydrofuran (THF), and even radical polymerization gives polymers rich in isotacticity. ${ }^{1,2}$ Poly(TrMA) obtained with $n$-BuLi in THF at $-78^{\circ} \mathrm{C}$ can be resolved into two optically active (+)- and (-)-polymers by liquid chromatography on optically active insoluble poly(TrMA). ${ }^{3}$ In addition, optically active polymers have been obtained directly by asymmetric polymerization with chiral anionic initiators, such as $(-)$-sparteine $((-)-\mathrm{Sp})-n-\mathrm{BuLi}^{4}{ }^{4} \quad(-)-(2 R, 3 R)-$ or $(+)-$ ( $2 S, 3 S$ )-2,3-dimethoxy-1,4-bis(dimethylamino)butane (DDB)-lithium amide, ${ }^{5}$ and DDB-fluorenyllithium (FILi) complexes. ${ }^{6}$ The chirality of the polymers is attributed to the stable helical conformation (atropisomerism) prevailing in a one screw sense. Bulky ester groups restrict uncoiling of the helical polymer chain which is produced through the

* Present address: Department of Applied Chemistry, Faculty of Engineering, Kagoshima University, Korimoto, Kagoshima 890, Japan.

** To whom all correspondences should be addressed. 
polymerization reaction. In the case of TrMA, the optically active polymer with nearly $100 \%$ one-handed helicity has been already obtained in the polymerization with these chiral lithium complexes., 5 On the other hand, these chiral complexes showed almost no enantiomer selectivity in the polymerization of less bulky racemic 1-phenylethyl methacrylate and 1,2-diphenylethyl methacrylate and gave atactic polymers. ${ }^{8}$ Highly enantiomer-selective polymerization has been observed in the polymerization of several racemic methacrylates such as 1-phenylethyl methacrylate and 1,2-diphenylethyl methacrylate with (-)-sparteine-Grignard reagent complexes in toluene at $-78^{\circ} \mathrm{C}$ and gave nearly $100 \%$ isotactic polymers. ${ }^{9-11}$ However, these Grignard complexes could not initiate the polymerization of TrMA and other bulky triarylmethyl methacrylates. ${ }^{1,8}$ These results urged us to investigate the asymmetric and enantiomer-selective polymerization of a bulky racemic monomer, phenyl-2-pyridyl-otolylmethyl methacrylate (PPyTMA) with the chiral lithium initiators to see the possibility of the enantiomer selection by the growing chain end with a rigid one-handed helical structure.
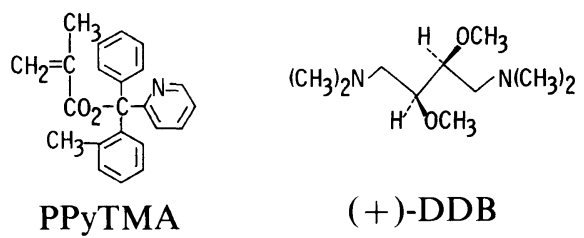

(+)-DDB

\section{EXPERIMENTAL}

\section{Materials}

Synthesis of PPyTMA. 2-Benzoylpyridine $(57.8 \mathrm{~g}, 0.32 \mathrm{~mol})$ dissolved in diethyl ether was added dropwise to $o$-tolylmagnesium bromide $(0.39 \mathrm{~mol})$ in diethyl ether $(400 \mathrm{ml})$ with vigorous stirring under nitrogen atmosphere. The reaction mixture was stirred for one hour after the completion of addition, and decomposed with saturated aqueous ammonium ch- loride. The ethereal solution was decanted and the residue was washed with diethyl ether. The combined ether solution was dried over magnesium sulfate. After the removal of the solvent, a yellow solid was obtained. This was recrystallized from methanol to give phenyl-2pyridyl-o-tolylmethanol. Yield $99 \mathrm{~g}(80 \%) ; \mathrm{mp}$ 105.4-106.5 $\mathrm{C}^{\circ}$ (lit. $^{12} 95-96^{\circ} \mathrm{C}$ ).

The carbinol $(33.5 \mathrm{~g}, 0.12 \mathrm{~mol})$ was converted to sodium alkoxide with sodium hydride $(5.6 \mathrm{~g}, 0.14 \mathrm{~mol})$ at $0^{\circ} \mathrm{C}$ in dry $\mathrm{THF}$ $(335 \mathrm{ml})$ under dry nitrogen. Then methacryloyl chloride $(14 \mathrm{ml}, 0.14 \mathrm{~mol})$ was added dropwise to the suspended alkoxide solution at $0^{\circ} \mathrm{C}$. After $2 \mathrm{~h}$, the mixture was heated at $60^{\circ} \mathrm{C}$ for $1 \mathrm{~h}$ and left overnight at room temperature under stirring. Diethyl ether and ice/water were added to the mixture, and the separated organic layer was collected. The ethereal layer was washed with saturated aqueous sodium bicarbonate and water, and then dried over magnesium sulfate. After ether was evaporated, the residue was recrystallized twice from diethyl ether and once from hexane. Yield $16.5 \mathrm{~g}(40 \%) ; \mathrm{mp} 113.8-114.5^{\circ} \mathrm{C} .{ }^{1} \mathrm{H}$ $\mathrm{NMR}\left(\mathrm{CCl}_{4}, 35^{\circ} \mathrm{C}\right.$, TMS $), 1.92\left(\mathrm{~s}, 3 \mathrm{H}, o-\mathrm{CH}_{3}\right)$, $2.00\left(\mathrm{~s}, 3 \mathrm{H}, \mathrm{CH}_{3}\right), 5.58$ and $6.26(\mathrm{~m}, 2 \mathrm{H}$, $\left.\mathrm{CH}_{2}=\mathrm{C}\right), 6.8-8.6 \mathrm{ppm}(\mathrm{m}, 13 \mathrm{H}$, aromatic$\mathrm{H})$; IR (nujol), $1725 \quad(\mathrm{C}=\mathrm{O}), 1635 \mathrm{~cm}^{-1}$ $(\mathrm{C}=\mathrm{C})$. Anal. Calcd for $\mathrm{C}_{23} \mathrm{H}_{21} \mathrm{O}_{2} \mathrm{~N}: \mathrm{C}$, $80.44 \%$; H, 6.16\%; N, $4.08 \%$. Found: C, $80.61 \%$; H, $6.17 \%$; N, $4.08 \%$.

Fluorenyllithium (FlLi) was synthesized by the reaction of fluorene and an equimolar amount of $n$-BuLi in toluene. (-)-DDB (Aldrich) was dried over calcium hydride and distilled under reduced pressure; $[\alpha]_{D}^{25}-13.1^{\circ}$ (toluene). Toluene was purified in the usual manner, mixed with a small amount of $n-\mathrm{BuLi}$, and distilled under high vacuum just before use.

\section{Polymerization Procedure}

Polymerization was carried out in a dry glass ampule under dry nitrogen. PPyTMA $(1.0 \mathrm{~g}, 2.9 \mathrm{mmol})$ was dissolved in toluene 
$(20 \mathrm{ml})$ and cooled to $-78^{\circ} \mathrm{C}$. The (-)-DDB-FlLi complex, which was prepared by mixing FlLi with 1.2 equivalent ( - -DDB in toluene at room temperature just before use, was then added to the monomer solution with a syringe; the molar ratio of monomer to FlLi was $20: 1$. The polymerization was terminated by the addition of a small amount of methanol and the reaction mixture was poured in a large amount of methanol. After separation of the polymer by centrifugation, methanol was evaporated as quickly as possible and the residue was extracted with benzene to recover the unreacted monomer. The polymer was extracted with THF to separate it into soluble and insoluble fractions. In order to determine the tacticity and molecular weight of the original polymer, poly(PPyTMA) was hydrolyzed in methanol containing a small amount of hydrochloric acid, and converted to poly(methyl methacrylate) (PMMA) by the methylation with diazomethane.

\section{Determination of Enantiomeric Excess of \\ Unreacted Monomer and Polymer}

The enantiomeric excess (e.e.) of unreacted monomer was determined by highperformance liquid chromatography (HPLC) using cellulose tris ( $p$-trifluoromethylphenylcarbamate) or cellulose tris(3,5-dichlorophenylcarbamate) coated on macroporous silica gel as a chiral stationary phase. ${ }^{13,14}$ The specific rotation $[\alpha]^{25}$ of the optically pure (+)-PPyTMA which was resolved by HPLC was estimated to be $328.2^{\circ}$ at $365 \mathrm{~nm}$ and $88.6^{\circ}$ at $589 \mathrm{~nm}$ in benzene $(c=0.94$ $\left.\mathrm{g} \mathrm{dl}^{-1}\right)$.

The e.e. of the polymer, which means the e.e. of polymerized monomer, can be estimated by calculation with polymer yield and the e.e. of unreacted monomer if the side reaction is negligible. $^{15}$ In the polymerization of PPyTMA, a small amount of methanolsoluble oligomer was detected when polymer yield was below $40 \%$. The yield of the oligomer was determined from ${ }^{1} \mathrm{H}$ NMR spectrum of the methanol soluble part, and this was added to polymer yield in the calculation.

\section{Measurements}

HPLC analysis was carried out with a chiral column $(25 \times 0.46$ (i.d.) $\mathrm{cm})$ on a JASCO TRIROTAR-II chromatograph equipped with a JASCO UVIDEC-100-III and DIP-181-C polarimetric detectors at $25^{\circ} \mathrm{C}$, hexane-2propanol $(95: 5$ or $90: 10(\mathrm{v} / \mathrm{v}))$ being used as an eluent. Gel permeation chromatography (GPC) was performed on a GPC column $(60 \mathrm{~cm} \times 2.1$ (i.d.) $\mathrm{cm})$ with maximum porosity of 3000 using chloroform $\left(3.0 \mathrm{ml} \mathrm{min}^{-1}\right)$ as an eluent. Optical rotation was measured on a JASCO DIP-181 polarimeter at $25^{\circ} \mathrm{C}$. ${ }^{1} \mathrm{H}$ NMR spectra were measured on a JNM-MH$100(100 \mathrm{MHz})$ spectrometer and tetramethylsilane was used as internal standard. IR spectra were recorded on a JASCO IR-810 infrared spectrophotometer. Circular dichroism (CD) spectra were measured on a JASCO-J40 CD apparatus equipped with a computerized data processor.

\section{RESULTS AND DISCUSSION}

\section{Enantiomer-Selective Polymerization of Race- mic PPyTMA}

Figure 1 shows a chromatogram of the resolution of racemic PPyTMA monomer on an HPLC column packed with cellulose tris(3,5-dichlorophenylcarbamate) coated on macroporous silica gel. PPyTMA was completely resolved into two antipodes giving a separation factor $\alpha=2.01$. $^{14}$ This efficient resolution allowed us to determine the e.e. of the unreacted monomer directly from chromatograms.

Tables I and II list the results of the enantiomer-selective polymerization of racemic PPyTMA with (-)-DDB-FlLi and (+)-DDB- $N, N^{\prime}$-diphenylethylenediamine monolithium amide (DPEDA-Li) complexes, respectively, in toluene at $-78^{\circ} \mathrm{C}$. Both the polymer and unreacted monomer were opti- 


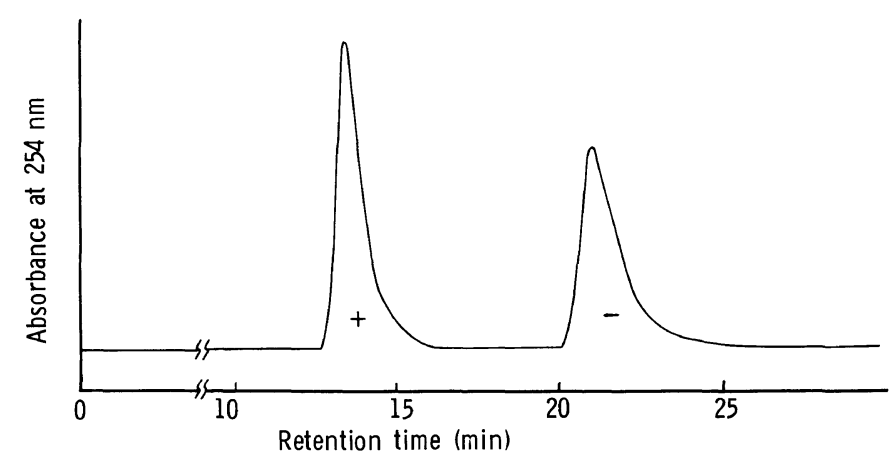

Figure 1. Resolution of the racemic PPyTMA on a cellulose tris(3,5-dichlorophenylcarbamate) column: column, $25 \times 0.46$ (i.d.) cm; eluent, hexane-2-propanol ( $95: 5$ ); flow rate, $0.5 \mathrm{ml} \mathrm{min}^{-1}$; temperature, $25^{\circ} \mathrm{C}$.

Table I. Enantiomer-selective polymerization of PPyTMA with (-)-DDB-fluorenyllithium complex in toluene at $-78^{\circ} \mathrm{C}^{\mathrm{a}}$

\begin{tabular}{|c|c|c|c|c|c|c|c|c|c|c|}
\hline \multirow{4}{*}{ No. } & \multirow{4}{*}{$\frac{\text { Time }}{\mathrm{h}}$} & \multirow{2}{*}{\multicolumn{2}{|c|}{ Yield $/ \%$}} & \multirow{4}{*}{$\%$ e.e. ${ }^{\mathrm{c}}$} & \multirow{4}{*}{$D P^{d}$} & \multicolumn{2}{|c|}{ THF-soluble polymer } & \multirow{4}{*}{$\begin{array}{c}\begin{array}{c}\text { THF-insoluble } \\
\text { polymer } \\
\text { yield }\end{array} \\
\%\end{array}$} & \multirow{2}{*}{\multicolumn{2}{|c|}{$\begin{array}{l}\text { Unreacted } \\
\text { monomer }\end{array}$}} \\
\hline & & & & & & \multirow{2}{*}{ Yield } & \multirow{3}{*}[\alpha]{$_{365}^{25} \mathrm{e}^{\mathrm{e}}$} & & & \\
\hline & & Polymer & Oligomer $^{b}$ & & & & & & {$[\alpha]_{365}^{25 \mathrm{f}}$} & $\%$ e.e. ${ }^{\mathrm{g}}$ \\
\hline & & & & & & $\%$ & & & & \\
\hline 1 & 0.05 & 7.2 & 6 & 7.2 & - & 7.2 & -273 & 0 & - & 1.1 \\
\hline 2 & 0.1 & 14.4 & 5 & 7.1 & - & 14.4 & -366 & 0 & - & 1.7 \\
\hline 3 & 0.15 & 17.0 & 4 & 10.2 & - & 17.0 & -375 & 0 & - & 2.7 \\
\hline 4 & 0.23 & 22.3 & 3 & 25.1 & - & 22.3 & -193 & 0 & - & 8.5 \\
\hline 5 & 0.33 & 23.5 & 3 & 27.7 & - & 23.5 & -42 & 0 & $+6^{\mathrm{h}}$ & 10.0 \\
\hline 6 & 0.50 & 28.2 & 2 & 31.4 & 12 & 28.2 & -55 & 0 & $+25^{\mathrm{h}}$ & 13.6 \\
\hline 7 & 0.42 & 32.2 & 2 & 30.8 & - & 32.2 & +61 & 0 & $+36^{\mathrm{h}}$ & 16.0 \\
\hline 8 & 1.22 & 50.6 & 0 & 25.7 & - & 49 & +400 & 2 & +86 & 26.3 \\
\hline 9 & 1.73 & 57.4 & 0 & 24.5 & 16 & 53 & +467 & 4 & +104 & 33.0 \\
\hline 10 & 3.50 & 68.9 & 0 & 19.5 & - & 62 & +647 & 7 & +142 & 43.2 \\
\hline 11 & 3.07 & 75.0 & 0 & 17.8 & - & 65 & +476 & 10 & +175 & 53.4 \\
\hline 12 & 4.25 & 79.5 & 0 & 13.9 & - & 69 & +250 & 11 & +180 & 53.9 \\
\hline 13 & 5.0 & 82.3 & 0 & 11.3 & - & 69 & +277 & 13 & +172 & 52.4 \\
\hline 14 & 6.0 & 87.9 & 0 & 7.3 & - & 70 & +248 & 18 & +173 & 52.8 \\
\hline 15 & 24 & 98.0 & 0 & - & 29 & 80 & +76 & 18 & - & - \\
\hline
\end{tabular}

a PPyTMA $1 \mathrm{~g}$, toluene $20 \mathrm{ml}$, [PPyTMA]/[FlLi] $=20$.

b Yield of oligomer soluble in methanol.

c Calculated e.e. of polymerized monomer.

d Determined by GPC of PMMA derived from poly(PPyTMA).

e In THF. ${ }^{f}$ In benzene. $\mathrm{g}$ Determined by chiral HPLC.

${ }^{\mathrm{h}}$ Included oligomer soluble in methanol.

cally active and $(-)$-enantiomer was preferentially polymerized over $(+)$-enantiomer when (-)-DDB was used as chiral ligand. The absolute configuration of the optical isomers is unknown at this moment. All the polymer were highly isotactic; for example, the tacticity of the polymer (No. 15 in Table I) was I : $\mathrm{H}: \mathrm{S}=94: 4: 2$. Since the methanol soluble part recovered in the early stage of the polymerization contained the oligomer of negative 
Table II. Enantiomer-selective polymerization of PPyTMA with (+)-DDB-DPEDA-Li complex in toluene at $-78^{\circ} \mathrm{C}^{\mathrm{a}}$

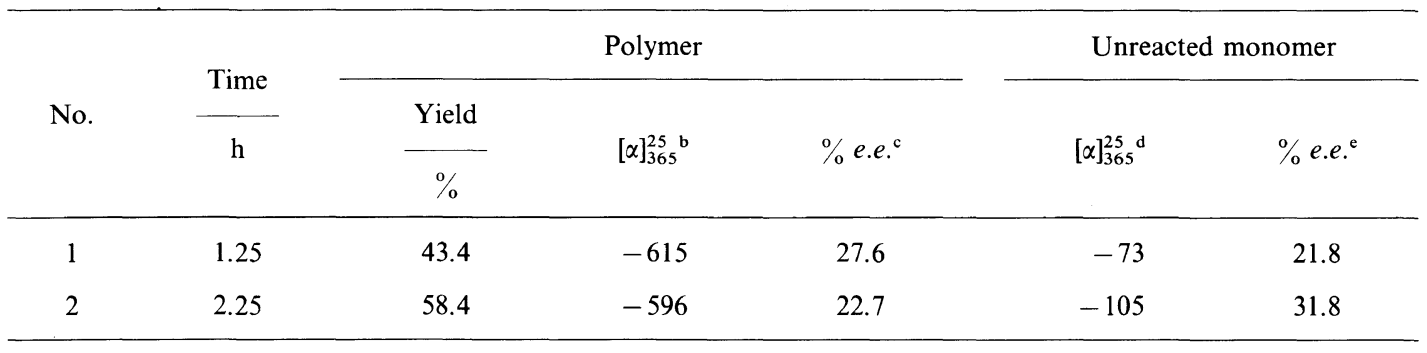

a PPyTMA $1 \mathrm{~g}$, toluene $20 \mathrm{ml}$, [PPyTMA] $/[\mathrm{Li}]=20$.

${ }^{b}$ In $\mathrm{CHCl}_{3}-2,2,2$-trifluoroethanol (9:1).

c Calculated e.e. of the polymerized monomer.

${ }^{\mathrm{d}}$ In benzene.

${ }^{e}$ Determined by chiral HPLC.

optical rotation and unreacted monomer, its specific rotation was lower than the value expected from the e.e. of unreacted monomer (No. 5, 6, and 7 in Table I). No side products were clearly present in the methanol soluble part. The optical rotation of the polymer in Table I varied in a complex manner as the polymerization proceeded, although all polymers were rich in (-)-PPyTMA units. The polymers obtained in the early stage of the polymerization showed negative rotation, and the ones obtained in higher polymer yields showed positive rotation.

Figure 2 shows the $C D$ spectra of $(+)$ - and (-)-poly(PPyTMA) and optically pure $(+)$ PPyTMA monomer in THF. The CD spectrum of the negative polymer (No. 6 in Table I) rich in (-)-PPyTMA was nearly the mirror image of that of $(+)$-PPyTMA in the region of $270-290 \mathrm{~nm}$. The low CD intensity of $(-)-$ poly(PPyTMA) must be due to the low e.e. of the polymer and the discrepancy below $260 \mathrm{~nm}$ may be ascribed to the existence of a small amount of higher-molecular-weight (+)poly(PPyTMA). On the contrary, the positive polymer (No. 9 in Table I), although rich in (-)-PPyTMA unit, showed a completely different spectrum. This indicates that the negative rotation of (-)-poly(PPyTMA) may be due to the chirality of asymmetric ester group

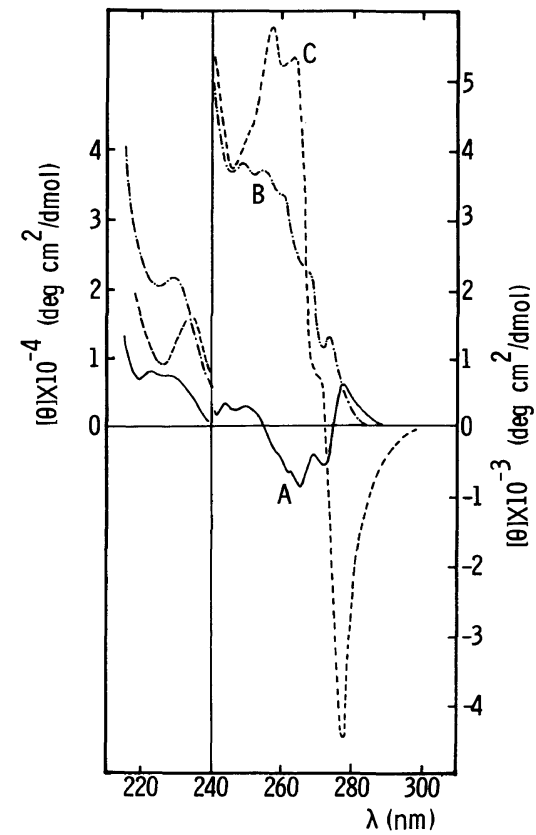

Figure 2. CD spectra of (-)-poly(PPyTMA) (No. 6 in Table I) (A), (+)-poly(PPyTMA) (No. 9 in Table I) (B), and optically pure $(+)$-PPyTMA monomer $(C)$ in THF.

of (-)-PPyTMA units incorporated preferentially in the polymer chain in the early stage of the polymerization and the positive rotation may be attributed to the chirality of the ordered conformation formed after the polymer grows to a certain degree of polymerization (DP). 


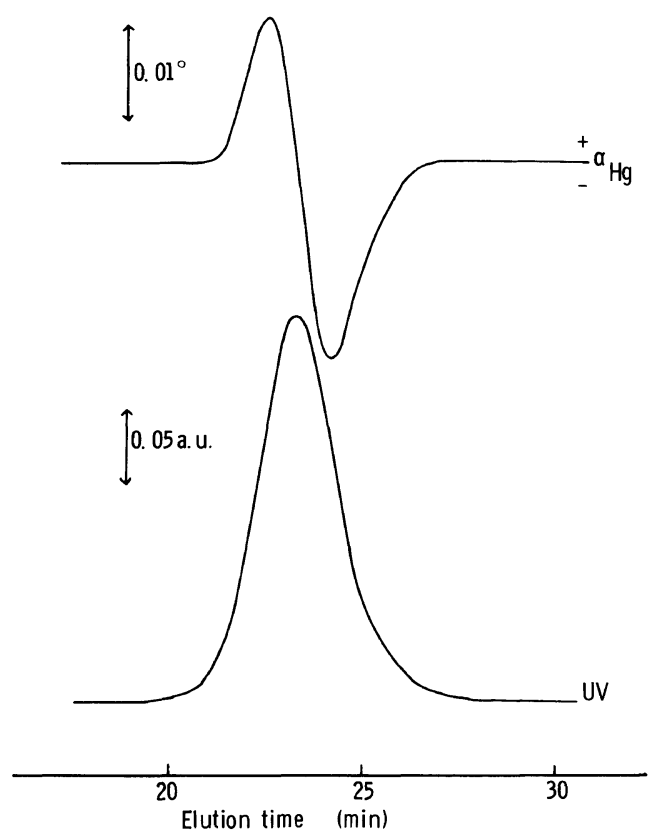

Figure 3. GPC curve of poly(PPyTMA) $\left([\alpha]_{365}^{25}-55^{\circ}\right.$; No. 6 in Table I).

Figure 3 shows the GPC curve of poly(PPyTMA) $\left([\alpha]_{365}^{25}-55^{\circ}\right.$; No. 6 in Table I) monitored with UV and polarimetric detectors. Although a UV detector showed a single GPC peak, a polarimetric detector showed a positive peak at higher-molecular-weight region and a negative one at lower-molecularweight region. The DP of PMMA derived from this poly(PPyTMA) was about 12 as shown in Table I. This seems to indicate that the poly(PPyTMA) takes on either right- or left-handed helical structure slightly before $\mathrm{DP}=12$ and the sign of optical rotation of the polymer changes from negative to positive rotations with the formation of the helix, even if rich in (-)-PPyTMA units.

The optical rotation of the THF-soluble polymer was maximum at about $70 \%$ polymer yield and decreased with a further increase in polymer yield. In the latter part of the polymerization, (+)-PPyTMA will be incorporated predominantly into the polymer because of its higher concentration over (-)-PPyTMA. This

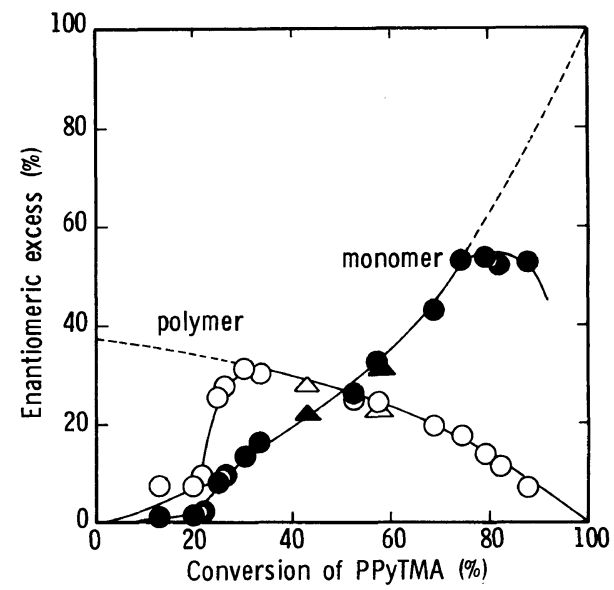

Figure 4. Plots of e.e. of polymer and unreacted monomer against conversion of PPyTMA in the polymerization with $(-)$-DDB-FlLi $(O, 0)$ and $(+)$ DDB-DPEDA-Li $(\triangle, \boldsymbol{\Delta})$ complexes in toluene at $-78^{\circ} \mathrm{C}$.

may lead to the formation of the monomer sequences which show large negative optical rotation.

Figure 4 illustrates the change of the enantiomeric excess of the polymer and unreacted monomer in the polymerization of PPyTMA with (-)-DDB-FlLi and (+)-DDBDPEDA-Li complexes. Both chiral initiators show the same enantiomer selectivity. In the early stage of the polymerization the enantiomer selectivity was very low and increased with the progress of the polymerization. This is a quite different result from the enantiomerselective polymerization of less bulky methacrylates in which the e.e. of the polymers is the highest in the early stage of the polymerization. $^{9-11,16}$ The increase of enantiomer selectivity in the early stage of the polymerization seems to be attributed to the formation of the one-handed helical structure of the polymer chain. Formation of such a rigid chiral helix may enhance the enantiomer selection. In the polymerization of less bulky racemic methacrylates such as 1-phenylethyl and 1,2-diphenylethyl methacrylate with the same chiral initiators systems, enantiomer selection was not observed and the polymers 
Table III. Enantiomer-selective copolymerization of PPyTMA $\left(M_{1}\right)$ with $\mathbf{M}_{2}$ by (-)-DDB-FlLi complex in toluene at $-78^{\circ} \mathrm{C}^{\mathrm{a}}$

\begin{tabular}{|c|c|c|c|c|c|c|c|c|c|}
\hline \multirow{4}{*}{$\mathbf{M}_{2}$} & \multirow{4}{*}{$\frac{\mathrm{M}_{1}}{\mathrm{M}_{1}+\mathrm{M}_{2}}$} & \multirow{4}{*}{$\frac{\text { Time }}{\min }$} & \multirow{2}{*}{\multicolumn{2}{|c|}{ Yield $/ \%$}} & \multicolumn{3}{|c|}{ Polymer } & \multirow{2}{*}{\multicolumn{2}{|c|}{ Unreacted monomer }} \\
\hline & & & & & \multirow{3}{*}{$\frac{\mathrm{M}_{1}{ }^{\mathrm{c}}}{\mathrm{M}_{1}+\mathrm{M}_{2}}$} & \multirow{3}{*}[\alpha]{$_{365}^{25}{ }^{d}$} & \multirow{3}{*}{$\%$ e.e. ${ }^{\mathrm{e}}$} & & \\
\hline & & & Total & PРyTMA $^{\mathrm{b}}$ & & & & \multirow[t]{2}{*}[\alpha]{$_{365}^{25} \mathrm{f}$} & \multirow[t]{2}{*}{$\%$ e.e. ${ }^{\mathrm{g}}$} \\
\hline & & & & & & & & & \\
\hline \multirow[t]{2}{*}{ TrMA } & 0.52 & 30 & 34.9 & 43.2 & 0.65 & -383 & 27.6 & +71 & 21.5 \\
\hline & 0.47 & 60 & 74.6 & 76.9 & 0.50 & -642 & 13.2 & +160 & 48.8 \\
\hline 1,1-DPEMA & 0.50 & 37 & 54.6 & 37.0 & 0.33 & -21 & 13.5 & - & 7.9 \\
\hline \multirow{2}{*}{ BzMA } & 0.33 & 5 & 56.9 & 11.1 & 0.20 & -2 & 0 & 0 & 0 \\
\hline & 0.33 & 30 & 74.9 & 20.0 & 0.27 & -7 & 0 & 0 & 0 \\
\hline
\end{tabular}

${ }^{a}\left[M_{1}+M_{2}\right] /[\mathrm{Li}]=20 . \quad{ }^{b}$ Conversion of PPyTMA. ${ }^{c}$ Determined by elemental analysis. ${ }^{d}$ In THF.

e Calculated e.e. of the polymer. ${ }^{\mathrm{f}}$ In benzene. ${ }^{\mathrm{g}}$ Determined by chiral HPLC.

obtained were nearly atactic. $^{8}$

The e.e. of polymerized monomer at each stage of the polymerization was estimated from Figure 4 and the results are plotted against conversion of PPyTMA monomer (Figure 5). When the conversion of PPyTMA was less than $20 \%$, the e.e. of the polymerized monomer was very low probably because the chiral helical structure was not built up satisfactorily as discussed earlier, but it drastically increased up to about $25 \%$ conversion around which a very high enantiomer selection (nearly $100 \%$ ) was attained. Figure 5 also shows that the e.e. of the polymerized monomer decreased as the polymerization proceeded and opposite (+)-PPyTMA monomer was predominantly polymerized over (-)-PPyTMA after 50\% conversion. At the latter part of the polymerization, (+)-PPyTMA was more rapidly polymerized. This must lead to the unusual decreases of the e.e. of unreacted monomer in Figure 4.

\section{Enantiomer-Selective Copolymerization of}

\section{PPyTMA with Other Methacrylates}

For confirmation of the importance of the one-handed helical structure on the enantiomer selection, the copolymerization of PPyTMA with TrMA or other less bulky methacrylates such as 1,1-diphenylethyl

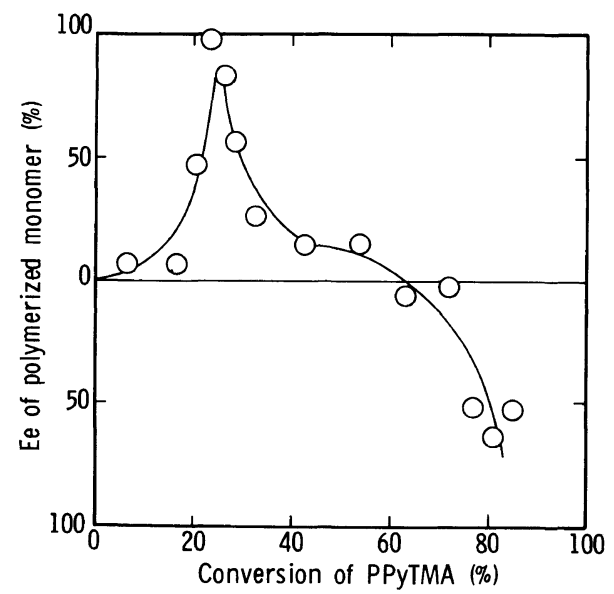

Figure 5. Plots of e.e. of polymerized monomer at each stage of the polymerization with (-)-DDB-FlLi complex against conversion of PPyTMA.

methacrylate (1,1-DPEMA) and benzyl methacrylate (BzMA) was investigated. 1,1DPEMA and BzMA formed optically inactive polymers in the polymerization with the present initiators. ${ }^{17}$ The results of the copolymerization with (-)-DDB-FlLi complex in toluene at $-78^{\circ} \mathrm{C}$ are summarized in Table III. The enantiomer selection was not influenced by the incorporation of TrMA and coisotactic polymer was formed, whereas in the copolymerization with 1,1-DPEMA, enantiomer selection decreased significantly, and isotac- 
ticity of the PMMA derived from the copolymer was very low (I:H:S=37:43:20). Furthermore, in the copolymerization with BzMA, no enantiomer selection was observed. These results also support that the enantiomer selection in the polymerization of PPyTMA is greatly due to the chiral conformation of a growing polymer chain, probably rigid one-handed helical structure.

\section{REFERENCES AND NOTES}

1. H. Yuki, K. Hatada, T. Niinomi, and Y. Kikuchi, Polym. J., 1, 36 (1970).

2. Y. Okamoto, M. Ishikura, K. Hatada, and H. Yuki, Polym. J., 15, 851 (1983).

3. Y. Okamoto, I. Okamoto, and H. Yuki, J. Polym. Sci., Polym. Lett. Ed., 19, 451 (1981).

4. Y. Okamoto, K. Suzuki, K. Ohta, K. Hatada, and H. Yuki, J. Am. Chem. Soc., 101, 4763 (1979).

5. Y. Okamoto, H. Shohi, and H. Yuki, J. Polym. Sci., Polym. Lett. Ed., 21, 601 (1983).

6. The complex gave $T H F-$ soluble poly $(\operatorname{TrMA})\left([\alpha]_{\mathrm{D}}^{25}\right.$ $-312^{\circ}$ ) quantitatively in the asymmetric polymerization of TrMA in toluene at $-78^{\circ} \mathrm{C}$. The polymer was fractionated with benzene-hexane $(1: 1(\mathrm{v} / \mathrm{v}))$ and insoluble part $(88 \%)$ showed $[\alpha]_{D}^{25}-345^{\circ}$.

7. Y. Okamoto, H. Mohri, M. Ishikura, and K. Hatada, J. Polym. Sci., Polym. Symp., 74, 125 (1986).

8. Y. Okamoto, E. Yashima, and K. Hatada, unpublished results.

9. Y. Okamoto, K. Urakawa, and H. Yuki, J. Polym. Sci., Polym. Chem. Ed., 19, 1386 (1981).

10. Y. Okamoto, K. Suzuki, T. Kitayama, H. Yuki, H. Kageyama, K. Miki, N. Tanaka, and N. Kasai, J. Am. Chem. Soc., 104, 4618 (1982).

11. Y. Okamoto, E. Yashima, K. Hatada, H. Yuki, H. Kageyama, K. Miki, and N. Kasai, J. Polym. Sci., Polym. Chem. Ed., 22, 1831 (1984).

12. F. J. McCarty, C. H. Tilford, and M. G. V. Campen, Jr., J. Am. Chem. Soc., 79, 472 (1957).

13. Y. Okamoto, M. Kawashima, and K. Hatada, J. Am. Chem. Soc., 106, 337 (1984).

14. Y. Okamoto, M. Kawashima, and K. Hatada, $J$. Chromatogr., 363, 173 (1986).

15. Y. Okamoto, H. Gamaike, and H. Yuki, Makromol. Chem., 182, 2737 (1981).

16. H. Suda, S. Kanoh, N. Murose, S. Goka, and M. Motoi, Polym. Bull., 10, 162 (1983).

17. When these two monomers were polymerized with (+)-DDB-FlLi complex in toluene at $-78^{\circ} \mathrm{C}$ in an optical cell, no optical rotation was observed at $-78^{\circ} \mathrm{C}$. The same was true in the polymerization with (-)-Sp-ethylmagnesium bromide complex which gave highly isotactic polymers. 